\title{
Can PRP effectively treat injured tendons?
}

James H-C. Wang

MechanoBiology Laboratory, Department of Orthopaedic Surgery, University of Pittsburgh

School of Medicine, Pittsburgh, PA, USA

\section{Corresponding author:}

James H-C. Wang

MechanoBiology Laboratory, Department of Orthopaedic Surgery, University of Pittsburgh

School of Medicine

210 Lothrop St, BST, E1640, Pittsburgh, PA

15213, USA

E-mail:wanghc@pitt.edu

\section{Summary}

PRP is widely used to treat tendon and other tissue injuries in orthopaedics and sports medicine; however, the efficacy of PRP treatment on injured tendons is highly controversial. In this commentary, I reason that there are many PRP- and patient-related factors that influence the outcomes of PRP treatment on injured tendons. Therefore, more basic science studies are needed to understand the mechanism of PRP on injured tendons. Finally, I suggest that better understanding of the PRP action mechanism will lead to better use of PRP for the effective treatment of tendon injuries in clinics.

KEY WORDS: tendon injuries, tendinopathy, $P R P$, orthopaedics, sports medicine.

Recent increases in the popularity of sports and the use of computers has created a rise in tendon injuries in both athletic and occupational settings. Tendon injuries, both acute and chronic (or tendinopathy), lower the quality of life of affected individuals and increase the costs of health care. Because injured tendons undergo a slow and incomplete healing process and often form scar tissue after healing, they are susceptible to re-injury ${ }^{1}$. Thus, restoring the normal structure and function to injured tendons remains one of the greatest challenges in orthopaedics and sports medicine.

In recent years, physicians in the orthopaedics and sports medicine field have adopted platelet-rich-plasma (PRP) to treat injured tendons and other tissues ${ }^{2}$.
PRP is the plasma fraction of the blood; it contains concentrated platelets and, in many cases, white blood cells (WBCs). PRP is autologous and therefore considered to be inherently safe; it provides a natural conductive scaffold, and is a reservoir of many growth factors (e.g. PDGF, TGF- $\beta$, VEGF, and HGF), which can enhance healing of injured tissues including tendons ${ }^{3,4}$. However, no study has thus far demonstrated the exclusive role of PRP growth factors in enhancing tissue healing, and the role of other molecules should also be considered ${ }^{5}$.

PRP has been shown to effectively treat acute and chronic tendon injuries ${ }^{6-9}$; however, little or no effect was also reported by others when PRP was used to treat similar conditions ${ }^{10-12}$. The current controversy over PRP's efficacy may be best addressed by basic science studies performed on cellular and animal models under well-controlled conditions. These models may include RNA silencing in vitro and gene knock-out or knock-down in mice in vivo to address specific functions of individual molecules contained in PRP. For example, a novel transgenic strategy has been recently developed to induce gene knock-down in platelets, which will allow characterization of genes involved in platelet production and the function of platelets in mice ${ }^{13}$.

In two of our recent studies, we investigated the effect of PRP on tendon stem/progenitor cells (TSCs) using a cell culture model ${ }^{14}$ and also determined the anti-inflammatory effects of PRP on tendon inflammation using both culture and animal models ${ }^{15}$. In the first study we aimed to test whether PRP treatment can promote tendon healing ${ }^{14}$. For this, we prepared PRP using methods that minimized the amounts of leukocytes and named it as the 'PRP-releasate'. Our results showed that treating TSCs with PRP-releasate induced TSC differentiation into active tenocytes, which proliferated quickly and produced abundant collagen, indicating the potential of PRP to enhance the repair of injured tendons. Additionally, PRP did not induce non-tenocyte differentiation into chondrocytes, adipocytes, or osteocytes ${ }^{16}$, suggesting that PRP treatment does not increase the risk of nontendinous tissue formation in treated tendons.

In the second study ${ }^{15}$, we demonstrated that PRP's anti-inflammatory function is mediated via HGF contained in PRP by suppressing the levels of prostaglandin biosynthetic pathway components (COX-1, COX-2, and mPGES-1 expression) and $\mathrm{PGE}_{2}$ production. These results were corroborated by our animal model studies where PRP injections (which may contain small amounts of leukocytes due to difficulty in removing all these cells from a small volume) re- 
duced COX-1 and COX-2 protein expression and lowered $P G E_{2}$ levels in the wounded Achilles tendons of mice. These results have clinical implications; high levels of $\mathrm{PGE}_{2}$ are known to cause pain ${ }^{17-19}$, decrease cell proliferation and collagen production ${ }^{20}$, cause aberrant differentiation of TSCs into non-tenocytes $^{21}$, and induce degenerative changes in rabbit tendons ${ }^{22}$. Therefore, PRP's ability to reduce PGE 2 production is expected to benefit the healing of injured tendons. Additionally, growth factors contained in PRP can stimulate tendon healing and the fibrin gel in PRP can serve as a natural scaffold to attract cells, thus resulting in enhanced tendon healing.

Many conflicting results have been reported on the efficacy of PRP treatment, which are due in part, to the various factors that influence the outcome of treatments in clinical settings. The most important among them are PRP- and patient-associated factors. The PRP-related factors include: 1) type of preparation, which varies significantly (some PRP preparations contain WBCs, which release inflammatory agents, such as IL-1 $\beta$ and TNF- $\alpha$ and hence may cause catabolic effects on treated tissues); 2) platelet concentration, which determines the amount of growth factors present in PRP preparations; higher concentration of platelets in PRP may not be necessarily optimal at least for cell proliferation ${ }^{23}$; 3) use of activated or non-activated PRP; non-activated PRP may promote tissue healing more efficiently than activated PRP24; 4) method of PRP activation (the use of calcium or thrombin may yield differential results); 5) mode of application (whether injection or implantation of PRP gel); and 6) frequency of PRP applications during treatment.

The patient-associated factors include: 1) age (PRP is more effective in young patients, who have more and better-quality stem cells); 2) type of tendon injury (acute or chronic); 3) type of tissue injured (tendons or other soft tissues); 4) patient activity level; 5) treatment history; and 6) post-recovery plans (with or without rehabilitation) ${ }^{25}$. Whether patients are in rehabilitation or resume daily activities after PRP treatment could also be beneficial or detrimental. This is because moderate mechanical loading is known to suppress cellular inflammation ${ }^{26}$ and induce anabolic changes in tendon cells, but excessive mechanical loading worsens cellular inflammation by increasing $\mathrm{PGE}_{2}$ production ${ }^{21}$ and inducing aberrant differentiation of TSCs into non-tenocytes, which may lead to degenerative tendinopathy ${ }^{16}$.

In current clinical settings, PRP is prepared by centrifugation and a pre-determined dose is used for all types of tissue injuries. This "one-size-fits-all" approach is sub-optimal and, not surprisingly, skews the outcome of PRP treatment. Recent studies have also shown that pro- and anti-angiogenic PRP components are segregated within granules and can be released selectively ${ }^{27}$, suggesting that the use of 'total' activated PRP to treat all types of tissue injuries may not be optimal. Therefore, selective release of specific molecular components within PRP preparations may improve healing outcomes.
Other varying factors frequently used in clinical studies are the various outcome measures of PRP treatment, including VAS (Visual Analog Scale), DASH (Disabilities of the Arm, Shoulder, and Hand), and VISA-A (Victorian Institute of Sport AssessmentAchilles) scale scores. While these scoring systems are necessary in patient PRP studies, they are based on patients' own assessment of pain intensity and tendon function as well and thus unavoidably subjective and highly variable. To compensate such large variability, studies should include a large population of patients to assess the efficacy of PRP treatment. However, the sample sizes in a number of patient studies have been relatively small $6,10,11$. Indeed, patient studies with greater statistical power as well as adequate outcome measures have been called for ${ }^{28,29}$.

As demonstrated by many previous studies and our own basic science research, PRP is likely a promising treatment to enhance the healing of injured tendons, but the factors mentioned above should be considered in current clinical practices and in the design of experimental research. This field would advance immensely with the optimization of PRP factors through additional basic science studies performed under well-controlled conditions ${ }^{30}$. We have linked at least one PRP component (HGF) to reduced inflammation and pain during tendon healing ${ }^{15}$. Additional studies that target various PRP components in vivo are needed to better understand their functions and enable the delivery of personalized PRP to patients. To ensure the efficacy of PRP treatment, guidelines for such treatment should be formulated based on the findings of basic science studies. It is anticipated that by optimizing PRP-associated factors and patient-related factors, we can effectively treat injured tendons in clinics.

\section{References}

1. Evans RB. Managing the injured tendon: current concepts. J Hand Ther. 2012;25:173-189.

2. Foster TE, Puskas BL, Mandelbaum BR, Gerhardt MB, Rodeo SA. Platelet-rich plasma: from basic science to clinical applications. Am J Sports Med. 2009;37:2259-2272.

3. Molloy T, Wang Y, Murrell G. The roles of growth factors in tendon and ligament healing. Sports Med 2003; 33:381-394.

4. Wang JH. Mechanobiology of tendon. J Biomech. 2006;39:1563-1582.

5. Andia I, Sanchez M, Maffulli N. Tendon healing and plateletrich plasma therapies. Expert Opin Biol Ther. 2010;10:14151426.

6. Sanchez M, Anitua E, Azofra J, Andia I, Padilla S, et al. Comparison of surgically repaired Achilles tendon tears using platelet-rich fibrin matrices. Am J Sports Med. 2007;35:245251.

7. Thanasas C, Papadimitriou G, Charalambidis C, Paraskevopoulos I, Papanikolaou A. Platelet-rich plasma versus autologous whole blood for the treatment of chronic lateral elbow epicondylitis: a randomized controlled clinical trial. Am J Sports Med. 2011;39:2130-2134.

8. Gaweda K, Tarczynska M, Krzyzanowski W. Treatment of Achilles tendinopathy with platelet-rich plasma. Int J Sports Med. 2010;31:577-583. 
9. Monto RR. Platelet rich plasma treatment for chronic Achilles tendinosis. Foot Ankle Int. 2012;33:379-385.

10. de Jonge S, de Vos RJ, Weir A, van Schie HT, Bierma-Zeinstra SM, et al. One-year follow-up of platelet-rich plasma treatment in chronic Achilles tendinopathy: a double-blind randomized placebo-controlled trial. Am J Sports Med. 2011;39:16231629.

11. de Vos RJ, Weir A, van Schie HT, Bierma-Zeinstra SM, Verhaar JA, et al. Platelet-rich plasma injection for chronic Achilles tendinopathy: a randomized controlled trial. JAMA. 2010;303: 144-149.

12. Schepull T, Kvist J, Norrman H, Trinks M, Berlin G, et al. Autologous platelets have no effect on the healing of human achilles tendon ruptures: a randomized single-blind study. Am J Sports Med. 2011;39:38-47.

13. Takiguchi M, James C, Josefsson EC, Carmichael CL, Premsrirut PK, et al. Transgenic, inducible RNAi in megakaryocytes and platelets in mice. J Thromb Haemost. 2010;8:2751-2756.

14. Zhang J, Wang JH. Platelet-rich plasma releasate promotes differentiation of tendon stem cells into active tenocytes. Am J Sports Med. 2010;38:2477-2486.

15. Zhang J, Middleton KK, Fu FH, Im HJ, Wang JH. HGF Mediates the Anti-inflammatory Effects of PRP on Injured Tendons. Plos One. 2013;8:e67303.

16. Zhang J, Wang JH. Mechanobiological response of tendon stem cells: implications of tendon homeostasis and pathogenesis of tendinopathy. J Orthop Res. 2010;28:639-643.

17. Smith WL. The eicosanoids and their biochemical mechanisms of action. Biochem J. 1989;259:315-324.

18. Narumiya S, Sugimoto $\mathrm{Y}$, Ushikubi F. Prostanoid receptors: structures, properties, and functions. Physiol Rev. 1999;79: 1193-1226.

19. Fiorucci S, Mencarelli A, Palazzetti B, Distrutti E, Vergnolle $\mathrm{N}$, et al. Proteinase-activated receptor 2 is an anti-inflammatory signal for colonic lamina propria lymphocytes in a mouse model of colitis. PNAS. 2001;98:13936-13941.

20. Cilli F, Khan M, Fu F, Wang JH. Prostaglandin E2 affects pro- liferation and collagen synthesis by human patellar tendon fibroblasts. Clin J Sport Med. 2004;14:232-236.

21. Zhang J, Wang JH. Production of PGE(2) increases in tendons subjected to repetitive mechanical loading and induces differentiation of tendon stem cells into non-tenocytes. J Orthop Res. 2010;28:198-203.

22. Khan MH, Li Z, Wang JH. Repeated exposure of tendon to prostaglandin-E2 leads to localized tendon degeneration. Clin J Sport Med. 2005; 15:27-33.

23. Graziani F, Ivanovski S, Cei S, Ducci F, Tonetti M, et al. The in vitro effect of different PRP concentrations on osteoblasts and fibroblasts. Clin Oral Implants Res. 2006;17:212-219.

24. Scherer SS, Tobalem M, Vigato E, Heit Y, Modarressi A, et al. Nonactivated versus thrombin-activated platelets on wound healing and fibroblast-to-myofibroblast differentiation in vivo and in vitro. Plast Reconstr Surg. 2012;129:46e-54e.

25. Sheth U, Simunovic N, Klein G, Fu F, Einhorn TA, et al. Efficacy of autologous platelet-rich plasma use for orthopaedic indications: a meta-analysis. J Bone Joint Surg Am. 2012;94:298-307.

26. Yang G, Im HJ, Wang JH. Repetitive mechanical stretching modulates IL-1beta induced COX-2, MMP-1 expression, and PGE2 production in human patellar tendon fibroblasts. Gene. 2005;363:166-172.

27. Italiano JE Jr, Richardson JL, Patel-Hett S, Battinelli E, Zaslavsky A, et al. Angiogenesis is regulated by a novel mechanism: pro- and antiangiogenic proteins are organized into separate platelet alpha granules and differentially released. Blood. 2008;111:1227-1233.

28. Maffulli N, Del Buono A. Platelet plasma rich products in musculoskeletal medicine: any evidence? Surgeon. 2012;10:148150.

29. Padulo J, Oliva F, Frizziero A, Maffulli N. Muscle, Ligaments and Tendons Journal. Basic principles and recommendations in clinical and field science research. MLTJ. 2013;4:250-252.

30. Del Buono A, Papalia R, Denaro V, Maccauro G, Maffulli N. Platelet rich plasma and tendinopathy: state of the art. Int J Immunopathol Pharmacol. 2011;24:79-83 